\title{
LA CRISIS DEL CORONAVIRUS TRAS EL PRIMER ESTADO DE ALARMA ${ }^{1}$
}

\author{
Juan José Solozábal Echavarría \\ Catedrático de Derecho Constitucional, \\ Universidad Autónoma de Madrid
}

Cómo citar este artículo / Citation: Solozábal Echavarría, J. J. (2021). La crisis del coronavirus tras el primer estado de alarma. Tudela Aranda, J. (coord.)

Estado Autonómico y covid-19,

Colección Obras colectivas, Fundación Manuel Giménez Abad, Zaragoza.

DOI: https://doi.org/10.47919/FMGA.OC21.0203

SUMARIO: I. EL TRATAMIENTO NORMATIVO DE LA CRISIS SANITARIA. EL VIEJO Y EL NUEVO ESTADO DE ALARMA - II. LA NUEVA NORMALIDAD: LA CRISIS DE LOS CONFINAMIENTOS O CIERRES PERIMETRALES - III. LA NUEVA NORMALIDAD Y LA COORDINACIÓN POR EL ESTADO DE LAS COMPETENCIAS SANITARIAS: GOBIERNO Y CONSEJO INTERTERRITORIAL DEL SISTEMA NACIONAL DE SALUD

I. EL TRATAMIENTO NORMATIVO DE LA CRISIS SANITARIA. EL VIEJO Y EL NUEVO ESTADO DE ALARMA

1.- La crisis del coronavirus está suponiendo "una viva prueba" para el sistema territorial constitucional. Pero a nadie se le oculta que las dificultades en abordar la actual situación no pueden imputarse en exclusiva a nuestro modelo autonómico, en el que hay evidentes deficiencias. Antes bien podríamos pensar que el tensionamiento territorial debe sus dificultades a factores, por decirlo así, externos o exógenos, que no pueden ser analizados aquí pero que

\footnotetext{
1 Se trata de la transcripción de mi intervención telemática en las Jornadas organizadas por la Fundación Giménez Abad y la Fundación Konrad Adenauer que tuvo lugar el día 28 de octubre de 2020, y que llevaba por título "El Estado Autonómico y la nueva normalidad".
} 
deben ser señalados. Hablamos de dificultades que tienen su origen en limitaciones del sistema representativo o el funcionamiento del sistema político en general -polarización, dificultad para el entendimiento, poca disposición para el acuerdo- o incluso de déficits de la propia moral pública de los ciudadanos, como su escasa disposición para el sacrificio por el bien común, sobreprima de los intereses personales, tensiones en nuestro pluralismo, sea territorial o también de tipo intergeneracional, ideológico, etc. La crisis sanitaria ha de abordarse, en suma, por un sistema territorial con dificultades, notoriamente de encaje entre tensiones centrífugas y necesidad de coordinación, pero en un contexto político también adverso y con una moral ciudadana que quizás no pasa por su mejor momento. Debe advertirse asimismo que mis observaciones, por cuya sumariedad y esquematismo pido disculpas, se deben insertar en el marco del foro en que se formulan, de modo que será interesante, al menos para su mejor comprensión, ponerlas en relación con otras intervenciones que la han precedido.

2.- Mi intervención va a referirse al "periodo de la nueva normalidad", esto es, el interregno entre los dos estados de alarma generales, desde el 21 de junio hasta el 25 de octubre. En concreto me centraré en la consideración de lo que llamaré la crisis de los confinamientos perimetrales y el Consejo Interterritorial de Salud, como exponentes respectivamente de las capacidades de las Comunidades Autónomas, y la utilización por parte del Estado central de instrumentos de coordinación. Nuestro sistema de descentralización, como forma federativa que es, supone un equilibro entre una dinámica territorial que insiste en el autogobierno como capacidad de adopción de medidas propias, también en el campo sanitario y de la salud, de una parte; y de otra, el reconocimiento de una actuación de signo centrípeto de coordinación y de dirección autónoma en esta materia.

Pero es necesario preliminarmente señalar algo sobre la problemática general del estado de alarma y su regulación normativa en el plano constitucional e inmediatamente legal. Considero efectivamente que es muy positiva "la constitucionalización del estado de alarma" en nuestra Norma Fundamental, frente a lo que ocurre en otros países como Alemania e Italia. Se incrementa así la normatividad constitucional, que llega a comprender la excepcionalidad, y además referida a situaciones de crisis no sólo política, sino diríamos de tipo sanitario, natural o socioeconómico. Estas deficiencias se han llenado en el 
caso alemán, donde las prevenciones en las situaciones de necesidad tienen su origen en la demonización de cualquier atisbo de influencia schmittiana, por las previsiones de las constituciones de los estados miembros, y en el caso italiano por una legislación que descansa en la anticipación constitucional de limitaciones al derecho de libre movimiento por exigencias de la salud y una concepción muy amplia de las habilitaciones del decreto-ley en manos del Gobierno, fácilmente puestas en funcionamiento en la crisis sanitaria².

De todos modos, "la regulación constitucional" especialmente en el caso del estado de alarma es "muy parca" -la restricción de los derechos fundamentales durante este periodo resulta por defecto de lo establecido en el artículo 55 CE que establece los derechos suspendibles durante los estados de excepción y de sitio-. Pero la Ley de 1981 de los Estados de Alarma, Excepción y Sitio (LOAES) a que se remite la Norma Fundamental tampoco añade mucho a la regulación constitucional. En realidad, está pensando más en los otros estados excepcionales, descontando además la legítima sospecha de que lo que interesaba al constituyente era cubrir las suspensiones individualizadas de derechos antiterroristas.

Hay que señalar además que la LOAES no se fija en la problemática territorial de la legislación excepcional, pues el Estado Autonómico en esa fecha de 1981 de la promulgación de la ley no se ha puesto a andar prácticamente.

La sumariedad del derecho de excepción tanto en el nivel constitucional como en el legal sí que plantea un problema en "la interpretación" y es el de que, aunque se trate de un derecho sobre situaciones odiosas y que por tanto ha de mover al intérprete a una posición restrictiva, también se refiere a una materia de natural dinámica que debe dejar un cierto margen de discrecionalidad en el entendimiento de su regulación, de modo que no podamos pensar que lo no previsto está prohibido ${ }^{3}$.

3.- Parto de "la corrección de la declaración del estado de alarma" (Real Decreto 463 de 14 de marzo de 2020, según las previsiones de la Ley de LO 4/1981, LOAES), que contempla expresamente el supuesto de una crisis sanitaria por epidemia. Estamos ante limitaciones de derechos y no

2 J. J. Solozábal, "Algunas consideraciones generales sobre el Estado de alarma" en P. Biglino y J. Durán, Los efectos horizontales de la covid-19 sobre el sistema constitucional: estudios sobre la primera oleada, Fundación Manuel Giménez Abad, Zaragoza, págs. 19 y 20.

3 "Algunas consideraciones generales sobre el Estado de Alarma", loc. cit., pág. 10. 
suspensiones, lo cual es obvio sobre todo en las últimas prórrogas. La diferencia entre estas dos figuras puede ser difícil, pero es posible. La suspensión es una privación temporal del derecho, obviamente de su ejercicio, no de su titularidad, que no se pierde en la situación de crisis. La limitación es una restricción no esencial de las facultades que comprende el derecho, de manera que su disposición no sea meramente nominal o supuesta. La diferenciación de circunstancias en las que se produce la suspensión o la limitación, esto es, la atención a los supuestos del estado de excepción o del estado de alarma, reparando en su origen o causa no deja de ser algo problemática4: de un lado, la crisis sanitaria puede devenir en crisis constitucional, y de otro la quiebra del orden público, entendido como situación en la que no pueden ser atendidos los servicios esenciales, puede originar el estado de alarma.

"Lo que se busca" con la declaración del estado de alarma es la centralización de los esfuerzos en la lucha contra la pandemia, que se entiende es inevitable, dadas las competencias de las Comunidades Autónomas en materia sanitaria. De modo que hay razones técnicas para proceder a la centralización, lo que no oculta también la conveniencia, en el nivel simbólico o político, de ofrecer una imagen de unidad política en la crisis sanitaria, que exige una respuesta unitaria.

Anotamos entonces que, por lo que hace a los efectos de la Declaración en relación con el derecho autonómico, se produce una centralización, y la subordinación de las autoridades autonómicas. El Derecho autonómico, no importa su rango, queda desplazado como consecuencia de la Declaración y no por la operación de la cláusula de prevalencia: "no estamos ante un conflicto competencial". Esta capacidad desplazatoria se refiere no sólo al Decreto, sino también a las órdenes dictadas en su ejecución durante el tiempo del estado de alarma.

4.- "Si se compara" el viejo Decreto de Declaración del estado de alarma de 14 de marzo y el nuevo de 25 de octubre llaman significativamente la atención diversas diferencias. Me fijo, brevemente, en cinco.

\footnotetext{
${ }^{4}$ M. Aragón, "Epílogo", en P. Biglino y J. Durán, Los efectos horizontales de la covid-19 sobre el sistema constitucional: estudios sobre la primera oleada, op. cit.
} 
A) El nuevo decreto, frente al anterior, es "descentralizador". Aparece claramente enmarcado en la "cogobernanza" como oportunidad para el protagonismo de las Comunidades Autónomas en la lucha contra la pandemia. La intervención de las Comunidades Autónomas es inevitable dadas sus competencias en materia sanitaria y la recuperación de las mismas, tras la finalización del estado de alarma. La cuestión es si esta actuación deja espacio al Gobierno para ejercer la coordinación que constitucionalmente le corresponde, toda vez que las Comunidades Autónomas no se limitan a cumplir su papel en sus respectivos territorios, sino que intervienen en la determinación de la política sanitaria nacional, como miembros del Consejo Interterritorial de Salud, un instrumento federal, cuyas funciones deben desempeñarse en términos constitucionalmente correctos, según se verá.

B) El Decreto contempla un nuevo supuesto de colaboración normativa en "círculos concéntricos": el derecho estatal es complementado por el derecho autonómico, pero no según la pauta básica en la que lo que corresponde al Estado es la fijación de los principios o criterios fundamentales en una materia, objeto de desarrollo por las Comunidades Autónomas. La pauta de la colaboración acostumbrada es vertical mientras que ahora la colaboración es horizontal y no según criterios de subordinación. Aunque esto se debe entender con cuidado: a veces las bases se ocupan de una cuestión concreta, si bien nodal; y en cualquier caso lo que queda para el desarrollo no son cuestiones accesorias o de detalle, como es sabido. Pero el contraste es claro: el derecho del Estado no es una norma de principios y regula el centro de la materia, hablemos de la libertad de movimientos o del derecho de reunión. Ahora la colaboración normativa no se produce entre normas del mismo rango: el supuesto recuerda la remisión normativa intraordinamental entre la ley y el reglamento, pero anotemos que aquí el reparto material se hace en términos de clara inferioridad del reglamento, lo que no es el caso en la colaboración interordinamental que nos ocupa. Las relaciones entre el Decreto y las disposiciones autonómicas permiten también ser consideradas desde la óptica de los supuestos contemplados en el artículo 150.2 CE, que será una cuestión de la que, como se verá, digamos algo de inmediato.

C) "Los destinatarios del Decreto" son los ciudadanos y las Comunidades Autónomas. Los términos en las que las Comunidades Autónomas quedan vinculadas por el Decreto ya han quedado tratados: en principio el conflicto 
entre el Derecho de Estado y el de las Comunidades Autónomas queda excluido, pues la relación entre ambos es de yuxtaposición y no de ocupación. Los ciudadanos quedarían sujetos a los dos órdenes normativos, teniendo en cuenta que, para la clarificación del Derecho autonómico, es indispensable el conocimiento del Derecho estatal, pues, como sabemos, aquel tiene sentido en los márgenes o como complemento de este.

D) Se trata de una norma muy "sumaria". Siendo dudoso, dada su escasa densidad, que se alcancen los estándares establecidos por el Tribunal Constitucional en relación con la limitación de derechos o de la Comisión de Venecia respecto de la legislación excepcional. Téngase presente que el decreto, que tiene rango de ley, abre el paso a limitaciones de derechos por parte de la autoridad delegada "a la vista de la evolución de los indicadores sanitarios, epidemiológicos, sociales, económicos y de movilidad"; y que la coordinación por parte del Gobierno para asegurar el cumplimiento de las medidas se atribuye, bajo la presidencia del Ministro de Sanidad, al Consejo Interterritorial del Sistema Nacional de Salud que adoptará cuantos acuerdos procedan, incluidos, en su caso, "el establecimiento de indicadores de referencia y criterios de valoración del riesgo". Ciertamente la "vaguedad" en el establecimiento de los supuestos en que procede la actuación limitadora de las Comunidades Autónomas es bien patente y la habilitación que se hace al Consejo para que establezca los supuestos de la actuación de coordinación es prácticamente en blanco, sin la asunción de cautela o condicionamiento alguno.

E) Los términos en que se contempla la actuación delegada de las Comunidades Autónomas no asegura el "engarce correcto" entre la normación estatal y la autonómica. En efecto se trata de una delegación peculiar en la medida que no se determina el tipo de reasignación competencial que permite, ni contempla con suficiente precisión los casos en que procede. Tampoco contempla la impartición de instrucciones por parte del delegante ni se preocupa por detallar los instrumentos de control y los efectos del mismo. Cabe preguntarse si la delegación contemplada en el decreto tiene que ver con los supuestos de traslación competencial que se contienen en el artículo 150.2 de la Constitución, que se refiere a la delegación o transferencia de facultades correspondientes a materia de titularidad estatal mediante ley orgánica. La comprensión de estas figuras puede intentarse desde la teoría constitucional y 
administrativa, pero mejor es atender a su utilización efectiva. Lo que enseñan los diversos ejemplos de Leyes de transferencias y delegación es que las Leyes de transferencias, reservan esta figura para la reasignación de competencias legislativas (por ejemplo en el caso de las hechas a favor de las Comunidades Autónomas que accedieron a la autonomía por la vía del artículo $143 \mathrm{CE}$ ), y solo ejecutivas en el caso de delegación de facultades del Estado a las Comunidades Autónomas (así en una materia como los transportes por carretera y cable). Ambas operaciones se llevan a cabo de acuerdo con instrucciones, aunque sean más detalladas en el caso de la delegación de facultades ejecutivas; y sometidas a control más alto y con mayores garantías en el caso de la transferencia 5 .

Como se ha visto hasta ahora, con lo que nos encontramos en la delegación del Decreto es con una habilitación casi en blanco ${ }^{6}$ para tomar decisiones que se instrumentan en normas reglamentarias de diverso tipo de las Comunidades Autónomas pero que van más allá de la simple ejecución, pues tienen un amplio margen discrecional, aunque sea con un carácter cumplimentador del decreto estatal de declaración del estado de alarma. El Decreto contempla "dos tipos de control": uno preventivo, consistente en la necesidad de comunicarse por parte de las autoridades delegadas las medidas limitadoras adoptadas al Ministerio de Sanidad (art. 9), comunicación que no equivale a solicitud de autorización ni prevé facultades suspensivas por parte de la autoridad delegante; y un control a posteriori parlamentario, imponiéndose al Ministro de Sanidad, en caso de prórroga, la comparecencia quincenal ante la Comisión de Sanidad y Consumo del Congreso de los Diputados para dar cuenta de la aplicación de las medidas previstas en el decreto (art. 14). Naturalmente en los parlamentos autonómicos, se ejercerá el control referente al desarrollo de la declaración del estado de alarma por los gobiernos respectivos de acuerdo con las previsiones normativas correspondientes en el nivel estatutario, reglamentario o legal.

La verificación del control no se facilita con la larga duración de la prórroga del estado de alarma, acordado por el Congreso para 6 meses, que no es un plazo

\footnotetext{
5 J.J. Solozábal, "Algunas observaciones al Informe del Consejo de Estado sobre la reforma constitucional", ahora en Pensamiento federal español y otros estudios autonómicos, Editorial lustel, Madrid 2019, págs. 256 y 257.

${ }_{6}^{6}$ Véanse las pertinentes observaciones de M. Aragón en el coloquio del día 28 de octubre en relación con la ponencia de $\mathrm{P}$. Biglino y la mía.
} 
que observe el principio de proporcionalidad, establecido en la LOAES y que obliga a todos los poderes públicos, comenzando por el Gobierno que solicita la prórroga y el Congreso que la aprueba. Un plazo de menor duración de la prórroga evita los riesgos de dispersión o exceso de heterogeneidad en que pueden incurrir las Comunidades Autónomas; y así se daría la oportunidad de recomponer la unidad en la actuación contra la pandemia, abordable también según criterios de flexibilidad que pueden producir una mayor eficacia, reparando la previsión abusiva de la Disposición final primera del Decreto que consiente su modificación mediante simple decreto, aunque comunicado, al Congreso, amparándose indebidamente en la LAOES $^{7}$. El artículo octavo dos de la Ley Orgánica 4/1981 de 1 de junio, como es sabido, no cubre propiamente la modificación de la declaración del estado de alarma, pues prevé más bien su desarrollo por esta vía reglamentaria, no su rectificación o ampliación ${ }^{8}$.

\section{LA NUEVA NORMALIDAD: LA CRISIS DE LOS CONFINAMIENTOS O CIERRES PERIMETRALES}

5.- Las Comunidades Autónomas, tras el término del estado de alarma, recuperan totalmente el ejercicio de sus competencias en sanidad, de acuerdo con el derecho sectorial sanitario, y que habían llevado a efecto antes de la Declaración. De este modo adoptan medidas que implican indudablemente "una intrusión en algún derecho fundamental".

Se trata de decretos, órdenes y puesta en marcha de sus planes de emergencia. Así se acuerdan: cierres perimetrales (Cataluña, Murcia, Extremadura, Andalucía); limitación de reuniones, con reducción de su aforo; restricciones en la frecuencia y ocupación de los transportes públicos; cierre de establecimientos públicos ${ }^{9}$.

\footnotetext{
7Disposición final primera del Decreto de Alarma 926/2020 de 25 de octubre: "Durante la vigencia del Estado de alarma declarado por este Real Decreto, el Gobierno podrá dictar sucesivos decretos qué modifiquen lo establecido en este, de los cuáles habrá de dar cuenta al Congreso de los diputados, de acuerdo con lo previsto en el artículo octavo dos de la Ley Orgánica 4/1981 de 1 de junio".
}

8 Art. 8.2 LOAES: "El Gobierno también dará cuenta al Congreso de los diputados de los decretos que dicte durante la vigencia del Estado de alarma en relación con este".

9 A. Nogueira, "Confinar el coronavirus. Entre el viejo derecho sectorial y el Derecho de excepción" El Cronista del Estado Social y Democrático, núm. 86-87, 2020, págs. 22 y ss. 
El derecho sectorial que se invoca es especialmente la Ley Orgánica de Medidas Especiales en materia de Salud Pública (LO 3/1983). Se trata de una Ley muy abierta que habilita a todas las Administraciones Públicas a tomar medidas invasivas de los derechos -así imponer cuarentenas y tratamientos-, que puede entenderse relacionada con la legislación infecciosa prevista en la LOAES.

Estamos ante decisiones que en buena parte coinciden con las tomadas en el decreto de Declaración del Estado de Alarma, aunque se trata de "medidas particulares en su ámbito personal y territorial" -en principio, por tanto, no indiscriminadas y generales-.

Estas medidas deben ser objeto, según el artículo 8 de la Ley de la Jurisdicción Contencioso Administrativa, de ratificación judicial, si implican privación o restricción de la libertad o de otro derecho fundamental.

La recuperación de las competencias sanitarias tras la finalización del estado de alarma no quiere decir que el Estado se desentienda de su ejercicio. A estos efectos es muy importante tener en cuenta el Decreto-Ley 21/2020, que se dicta en aplicación del Plan para la Transición hacia una Nueva Normalidad, acordado por el Consejo de Ministros el 28 de abril de 2020, por el que el Estado se reserva determinadas potestades de vigilancia e inspección toda vez que la crisis sanitaria no finaliza con la expiración del estado de alarma, aunque hayan dejado de necesitarse medidas extraordinarias de contención, incluidas las limitativas de la libertad de circulación, y que se orienta en el sentido de afianzar la coordinación, reforzando el funcionamiento del sistema nacional de salud. Este decreto no excluye la necesidad de que el Estado, con carácter excepcional y cuando así lo requieran motivos de extraordinaria gravedad o urgencia, adopte, con la colaboración de las Comunidades Autónomas, "cuantas medidas sean necesarias"; y contempla la modificación de la Ley de Cohesión y Calidad del Sistema Sanitario, para que el Consejo Nacional de Salud (art. 65) a través de la figura de las actuaciones coordinadas en salud pública, elabore o active planes y estrategias de actuación para afrontar emergencias sanitarias. En esta línea sin duda va 
también la modificación de la Ley de la Jurisdicción Contencioso-administrativa de septiembre de 2020, a la que luego nos referiremos ${ }^{10}$.

6.- El sistema parece funcionar sin especiales problemas, esto es, sin cuestionamiento serio ni político ni jurisdiccional, hasta lo que podríamos llamar "la crisis de los confinamientos o cierres perimetrales" (prohibición de entrar o salir de una población). Se trata de una restricción o limitación de un derecho (libertad de circulación); por tanto, no estamos ante la privación o perdida de una facultad ni de la suspensión de su ejercicio. Asimismo, nos referimos a una actuación de la Comunidad Autónoma fuera del estado de alarma, de modo que se pudiese alegar la delegación del Gobierno. Cierto que en esta restricción se va más allá de la previsión de la Ley de Medidas especiales en materia de Salud Pública, pues se trata, por su ámbito personal y territorial, "de una medida indiscriminada y general".

Ahora bien, la Ley de la Jurisdicción, reformada por la Ley 3/2020 de 28 de septiembre, estipula la ratificación judicial de supuestos de restricciones de los derechos fundamentales que no están contemplados personalmente. En efecto, dicha reforma extiende la autorización judicial a casos en los que los destinatarios de las restricciones de los derechos "no están identificados individualmente".

De otro lado, las medidas perimetrales pueden entenderse se toman en el marco habilitante de la Orden comunicada de actuaciones coordinadas de salud de 30 de septiembre de 2020 del Ministerio de Sanidad, según el artículo 65 de la Ley de Calidad y Cohesión del Sistema Sanitario. Esta Orden se cumplimenta por la Comunidad de Madrid; pero el Tribunal Superior no la convalida.

La decisión del TSJ de Madrid por Auto 128/220 negándose a la revalidación de las medidas, como decimos, tomadas por la Comunidad de Madrid en cumplimiento de la Orden del Ministerio, haciendo suyas las recomendaciones del Consejo Interterritorial de Salud, es bien discutible. Primero porque procede a examinar la regularidad (legalidad) de la Orden del Ministro de Sanidad, pues a su juicio el precitado artículo 65 no contempla la restricción de derechos

10 Me remito a la ponencia, presentada a estas jornadas, de A. Carmona, "El Estado Autonómico y las fases de la desescalada". 
fundamentales como acuerdo posible del Consejo Interterritorial. Sin embargo, tal control es claramente no pertinente, ya que debería haber correspondido a la Audiencia Nacional que examina la regularidad de las órdenes ministeriales o haber dado lugar al planteamiento de una cuestión de inconstitucionalidad ante el TC.

$\mathrm{Y}$, además, cuando menos, se ignora el principio curia novit iura, al indicarse la posibilidad por parte del Tribunal de alegarse como fundamento de la Orden de la Comunidad otra base legal, tal como sucedió cuando el mismo Tribunal confirmó los confinamientos por zonas básicas de salud de Madrid, deficiencia que bien pudo habérsele comunicado a la Comunidad de Madrid ${ }^{11}$. Este trámite hubiese sido especialmente indicado, al no haber en el procedimiento partes perjudicadas. De modo que el Tribunal debió haberse limitado a examinar la proporcionalidad de la medida de acuerdo con la base legal que se utilizaba.

La actuación ultra vires del Tribunal es más obvia en el caso del Tribunal Superior de Aragón (asunto cierre de la Almunia de Doña Godina) en su Auto 332/2020, cuando el Tribunal rechaza que haya base legal para decretar el confinamiento perimetral, según su interpretación de la Ley de Medidas, ignorando que una vez aprobada la Ley 3/2020, que expresamente prevé y regula la ratificación judicial de las decisiones sanitarias "generales", ya no cabe sostener que esas medidas no son posibles. El Tribunal elude la aplicación de la Ley de la Jurisdicción, sin cuestionar su constitucionalidad ${ }^{12}$.

Ante esta situación con toda pertinencia se ha suscitado la "conveniencia de una Ley", más allá de la Ley de medidas especiales que satisficiese los estándares de seguridad jurídica, en punto a la densidad y el rango, que exige nuestro Tribunal Constitucional en relación con las normas que estipulen restricciones de los derechos fundamentales. Sin duda las limitaciones de los derechos fundamentales de entidad, entre las que caen los cierres perimetrales y todavía más los confinamientos domiciliarios no totales, deben autorizarse

\footnotetext{
11 F. Velasco, Blog 8 de octubre. "El Tribunal de Justicia de Madrid no ratifica los confinamientos, por falta de autorización legal".

12 F. Velasco, Blog 11 de octubre, "También el TSJ de Aragón deniega la ratificación de medidas sanitarias (para La Almunia de Doña Godina)". Finalmente la Sección Primera de la Sala de lo Contencioso- Administrativo del Tribunal Superior de Aragón ha planteado una cuestión de inconstitucionalidad en relación con el artículo 10. 8 de la Ley de la Jurisdicción Contencioso- Administrativa, introducido por la disposición final segunda de la Ley de medidas procesales y organizativas para hacer frente a la Covid 19. El Tribunal Constitucional ha admitido a trámite la cuestión.
} 
por ley orgánica, y establecerse claramente los casos y vías en que dichas restricciones procedan.

Por lo que hace a "la intervención judicial" controlando los reglamentos con alcance restrictivo de los derechos fundamentales, que se contemplan en la Ley de la Jurisdicción, antes y después de su reforma, cabe hacer dos consideraciones. En primer lugar, y por lo que respecta a las órdenes de las Comunidades Autónomas, pueden entenderse como "una cautela centrípeta" en nuestro sistema autonómico, donde el control político parlamentario quizás no es muy operativo por el peso de los gobiernos. En segundo lugar, cabe preguntarse si ese recelo ante el control a priori judicial de la actividad reglamentaria no responde a un prurito un tanto doctrinario del entendimiento del principio de separación de poderes. Tengamos en cuenta que nuestro sistema es de separación no de división de poderes a ultranza y que en el mismo los jueces llevan a cabo una intervención preventiva de defensa de los derechos fundamentales ante peligros para los mismos procedentes de la Administración, aunque es cierto que individualizados, y no normativos. Nos referimos a las garantías jurisdiccionales cautelares o anticipadas en el caso del secreto de las comunicaciones o la inviolabilidad de domicilio. Del mismo modo, la dedicación exclusiva a la función jurisdiccional no impide que los jueces puedan integrar la administración electoral, con dedicación completa para el presidente de la Junta Electoral Central durante el período electoral, o, en su caso, la administración penitenciaria. En efecto, los jueces de vigilancia penitenciaria coadyuvan (no solo supervisan) en la función ejecutiva que corresponde también a la Administración penitenciaria (por ejemplo, autorizan los permisos de salida de los internos en determinados casos, y lo hacen ellos mismos, no en vía de recurso).

\section{LA NUEVA NORMALIDAD Y LA COORDINACIÓN POR EL ESTADO DE LAS COMPETENCIAS SANITARIAS: GOBIERNO $Y$ CONSEJO INTERTERRITORIAL DEL SISTEMA NACIONAL DE SALUD}

7.- Pero el Estado autonómico reconoce la sanidad como una competencia compartida en la que al Estado Central le corresponde la coordinación, según 
es sabido ${ }^{13}$. La coordinación sobre la sanidad es un título constitucional explícito (149.1.16 CE), junto a la de la política económica y la investigación científica y técnica (149.1.13 y 15 CE). Hay otros títulos competenciales implícitos, en realidad supraordenados al específico de la coordinación: artículo 97CE: dirección de la política del Estado, también de la sanitaria, por el Gobierno. $\quad \mathrm{Y}$ artículo 149 1.1 $\mathrm{CE}$ : aseguramiento de la igualdad constitucional -también el derecho a la salud- como competencia del Estado central.

La coordinación es una variedad de la colaboración que implica la dirección y preeminencia del Estado. Se trata de "una competencia obligatoria para el Estado", en el sentido de no disponible o facultativa, esto es, imprescindible o indefectible; "y obligatoria para las Comunidades Autónomas", en el sentido de vinculante, aunque normalmente se ejerza de modo voluntario. Da lugar a actuaciones coordinadas que deben ser eficaces, adecuadas y lo menos restrictivas posible de las atribuciones de las Comunidades Autónomas.

En el marco general de cooperación, operan instrumentalmente el Consejo Interterritorial del Sistema nacional de Salud y las demás Conferencias sectoriales. Aunque la cooperación se produce a través de otros instrumentos, que han sido utilizados con cierta profusión, a partir del momento de la declaración del estado de alarma. Me refiero a las Conferencias de Presidentes y múltiples Conferencias sectoriales bilaterales.

La caracterización correcta del Consejo Interterritorial de Salud y de sus acuerdos es capital, especialmente para ver si ha desempeñado correctamente su actuación y determinar si, en concreto, ofrece cobertura suficiente a limitaciones de derechos de parte de las Comunidades Autónomas.

El Consejo Interterritorial del Sistema Nacional de Salud es un "órgano administrativo auxiliar" del Gobierno, que es quien tiene a su cargo el diseño y control de la política sobre dicha materia; y adopta, consecuentemente por consenso, sus recomendaciones ${ }^{14}$. Lo que hace el Consejo, entonces, es conocer y debatir, dice la Ley de Cohesión y Calidad del Sistema Nacional de Salud, y no puede ser, además, de otro modo, puesto que el Consejo no es un

${ }_{13}$ Para lo que sigue, mi "El derecho constitucional a la salud en el Estado autonómico", ahora en Pensamiento federal y otros estudios autonómicos", lustel, Madrid 2019, págs. 281 y ss.

${ }_{14} \mathrm{Me}$ remito a la Ponencia presentada en estas jornadas por P. Biglino, con el título "Las competencias en el Estado Autonómico y su ejercicio". 
fundación

Manuel Giménez Abad

deEstudios Parlamentariosydel Estado Autonómico

órgano homogéneo. En efecto, se trata de un organismo qué está integrado por el Ministro y por los consejeros de sanidad de las Comunidades Autónomas, que designan a un vicepresidente; pero no es un órgano que tenga un sentir propio, que comparta una posición política.

"Las recomendaciones" del Consejo pueden informar las decisiones en materia sanitaria del Gobierno, pero no pueden sustituirlas 0 precederlas necesariamente. Finalmente, hemos de decir que es discutible que estas decisiones se trasladen mediante normas reglamentarias, de rango ministerial, esto es, órdenes; y no a través de decretos, teniendo en cuenta que pueden llegar a habilitar decisiones de las Comunidades Autónomas restrictivas de derechos. 